\title{
Effects of dietary Spirulina on antioxidant status, lipid profile, immune response and performance characteristics of broiler chickens reared under high ambient temperature
}

\author{
Sara Mirzaie ${ }^{1, *}$, Fahim Zirak-Khattab ${ }^{1}$, Seyed Abdollah Hosseini' ${ }^{2}$, and Hamid Donyaei-Darian ${ }^{3}$
}

* Corresponding Author: Sara Mirzaie Tel: +98-2156276639, Fax: +98-2156276605,

E-mail: smirzaie@irost.ir

' Department of Agriculture, Iranian Research Organization for Science and Technology (IROST), Tehran 3313193685, Iran

2 Department of Nutrition and Physiology, Animal Science Research Institute, Alborz 2146618261, Iran

${ }^{3}$ Pars Mobin Azma Laboratory, Guilan 41587444655, Iran

\section{ORCID}

Sara Mirzaie

https://orcid.org/0000-0003-1360-9109

Fahim Zirak-Khattab

https://orcid.org/0000-0003-4965-119X

Hamid Donyaei-Darian

https://orcid.org/0000-0002-1030-6084

Submitted Jun 27, 2017; Revised Jul 31, 2017; Accepted Aug 23, 2017
Objective: Spirulina has been recognized formerly as a filamentous spiral-shaped blue- green algae but more recently as a genus of photosynthetic bacteria (Arthrospira). This microorganism is considered as a rich source of essential nutrients for human and animals. The present study was conducted to determine potential application of Spirulina for heat- exposed broilers.

Methods: Two hundred and fifty Cobb 500 chicks with male to female in equal ratio with average initial weight of $615.6 \mathrm{~g}$ at 17 days of age were divided into 5 treatments with 5 replicates of 10 chicks. Treatment groups were as follows: positive and negative controls with $0 \%$ Spirulina supplement and three Spirulina receiving groups with $5 \mathrm{~g} / \mathrm{kg}(0.5 \%), 10 \mathrm{~g} / \mathrm{kg}(1 \%)$, and $20 \mathrm{~g} / \mathrm{kg}$ (2\%) supplementation. Spirulina receiving groups as well as positive control were exposed to high ambient temperature at $36^{\circ} \mathrm{C}$ for $6 \mathrm{~h} / \mathrm{d}$ from 38 to 44 days of age. Biochemical variables were measured in serum samples at $35,38,42$, and 45 days of broiler chickens age. Results: The results showed that supplementation of the diet with Spirulina decreased concentration of stress hormone and some serum lipid parameters while enhanced humoral immunity response and elevated antioxidant status whereas it didn't meaningfully affect performance characteristics. Nevertheless, feed conversion ratio was improved numerically but not statistically in broilers fed with $1 \%$ Spirulina under high ambient temperature.

Conclusion: Overall, the present study suggests that alleviation of adverse impacts due to high ambient temperature at biochemical level including impaired enzymatic antioxidant system, elevated stress hormone and lipid profile can be approached in broiler chickens through supplementation of the diet with Spirulina platensis.

Keywords: Antioxidant; Broilers; Heat Stress; Hypolipidemic Properties; Spirulina

\section{INTRODUCTION}

High ambient temperature is among the most prevalent environmental stress factors which negatively affect welfare, health and production of commercial poultry. Some of the important biochemical and physiological consequences of heat stress are reduced immunity function, increased production of free radicals and lipid peroxidation of cell membranes [1]. As a common environmental stressor, high ambient temperature creates higher demand for essential nutrients and antioxidants in the poultry's diet to reverse the impairments in health and performance of birds especially in intensive production systems [2]. Since efficient cooling of poultry housing is costly and to some extent impractical, many researches are interested in dietary manipulations to reduce the negative impact of heat stress on broilers. Many studies have reported that nutritional supplementations of vitamins and electrolytes [3,4], probiotics [5], prebiotics [6], organic acids and phytogenic [7], and some other feed additive or manage- 
ment practices $[2,8]$ are beneficial to attenuate the detrimental effects of environmental stress on performance of broiler chickens. At such a condition the natural feed additives such as algal biomass or extracts which are rich in antioxidants and other bioactive components may give dual advantage; first, helping birds to resist against inappropriate or stressful environmental condition and second, producing broilers with reduced level of cholesterol or total lipids and probably better antioxidant status.

Spirulina has been recognized formerly as filamentous spiral-shaped blue- green algae but more recently as a genus of photosynthetic bacteria (Arthrospira). This microorganism belongs to the class of Cyanophyta/Cyanobacteria that grow naturally in warm and alkaline aquatic media. Spirulina is considered a promising nutrient source due to its high protein content ( $65 \%$ to $70 \%$ dry matter) and great amount of vitamins, minerals and a wide variety of natural carotene and xanthophyll phytopigments [9]. Also, Spirulina is rich in other nutritionally beneficial organic molecules such as gamma linoleic acid, phycocyanins, phenolic acids, beta-carotene and chlorophyll [10].

Health benefits of Spirulina including antioxidant properties, hypolipidemic action and immunostimulating or anti- inflammatory effects $[11,12]$, have been verified by many researches using laboratory animals. In addition, the impacts of dietary Spirulina on domestic animal and poultry health and productivity under normal environmental conditions have been reported [13-15]. In a published work on evaluating Spirulina supplement in chickens under heat stress condition, Zeweil et al [16] demonstrated that Spirulina platensis could decrease adverse effects of chronic heat stress on growth performance and immunity of Gimmizah local strain of chicken.

Since to the best of our knowledge, limited information is available for the effects of microalgal species including Spirulina platensis as dietary supplement on broiler chickens under acute heat stress, the present study was planned to assess performance, immune response and antioxidant status of broiler chickens fed with such a nutritionally valuable natural supplement under the condition of high ambient temperature.

\section{MATERIALS AND METHODS}

\section{Experimental design}

The experiment was carried out according to the ethical guidelines for animal use and animal care of animal science research institute, Alborz province, where the experiment was performed. Two hundred and fifty Cobb 500 broiler chicks of mixed sex (male to female in equal ratio) with average initial weight of $615.6 \mathrm{~g}$ at 17 days of age were divided into five treatments with five replicates of 10 chicks. The birds were kept on floor pens $\left(3.25 \mathrm{~m}^{2} /\right.$ pen) with ad libitum access to feed and water. Diets were formulated by considering NRC [17] recommended levels to meet nutritional requirements. Treatment groups were as follows: positive and negative controls (ConP and ConN) with $0 \%$ supplementation and three Spirulina receiving groups with $5 \mathrm{~g} / \mathrm{kg}(0.5 \%), 10 \mathrm{~g} / \mathrm{kg}(1 \%)$, and $20 \mathrm{~g} / \mathrm{kg}$ (2\%) supplementation until the end of the experiment (day 45). The ingredients and compositions of the experimental diets are presented in Table 1. The birds were reared under normal environmental temperature until day 38 which was the onset of heat challenge. One week before the implementation of heat challenge, chickens in the ConN were moved to adjacent house with the same housing and management practices in order to continue to be reared in normal environmental temperature throughout the rearing period (last week temperature for ConN was $23^{\circ} \mathrm{C} \pm 1^{\circ} \mathrm{C}$ ). At day 38 , by using a jet heater in the house, Spirulina receiving groups as well as ConP were exposed to acute heat stress at $36^{\circ} \mathrm{C}$ for $6 \mathrm{~h} / \mathrm{d}$ until day 44 . After the heat challenge, excess heat was removed by evaporation cooling and increased ventilation. Chickens were weighted at day 17 and weekly afterward. Feed intake, feed conversion ratio (FCR) and possible mortality cases were also recorded weekly throughout the experiment.

\section{Sampling and measurements}

One of the consequences of high ambient temperature on broiler chickens is compromised most immune functions including antibody titers to sheep red blood cells (SRBC) [8]. To determine humoral immune response against SRBC, at days 31 and 38, one milliliter of a $10 \%$ suspension of SRBC in sterile phosphate buffered saline was injected intravenously in two birds from each replicate. One week following each injection, antibody titers were measure in separated sera by haemagglutination assay (HA) using 96-well plates as described previously [18].

At days 35, 38 (before the onset of heat challenge), 42 and 45 , blood samples were obtained from the brachial vein of two birds from each replicate. These samples were used to prepare sera to determine the activity of blood antioxidant enzymes including superoxide dismutase (SOD) and glutathione peroxidase (GPx), malondialdehyde (MDA), lipid parameters including total cholesterol, triglyceride, high- density lipoprotein cholesterol (HDL-C) and total lipid as well as corticosterone level. The activities of SOD and GPx were measured by methods previously described elsewhere $[19,20]$. MDA concentration was measured by thiobarbituric acid reactive substances assessment and expressed as $\mathrm{nmol} / \mathrm{mL}$. Concentrations of lipid parameters were determined using commercial kits with prepared reagents according to manufacturer instruction (Ziest Chem Co. Tehran, Iran). The concentration of corticosterone was determined using chicken corticosterone ELISA kit (ZellBio, GmbH, Ulm, Germany). Since the number of lymphocytes may be influenced under stressful conditions, heterophil to lymphocyte ratio $(\mathrm{H} / \mathrm{L})$ is also a common indicator of stress in animals and poultry. For deter- 
Table 1. The ingredients and composition of experimental diets

\begin{tabular}{|c|c|c|c|c|}
\hline \multirow{2}{*}{ Item } & \multicolumn{4}{|c|}{ Spirulina (\%) } \\
\hline & 0 & 0.5 & 1 & 2 \\
\hline \multicolumn{5}{|l|}{ Ingredients } \\
\hline Soybean meal & 30 & 29.5 & 29 & 28 \\
\hline $\mathrm{NaHCO}_{3}$ & 0.1 & 0.1 & 0.1 & 0.1 \\
\hline Salt & 0.3 & 0.3 & 0.25 & 0.3 \\
\hline DL-methionine & 0.13 & 0.13 & 0.13 & 0.13 \\
\hline L-lysine & 0.08 & 0.08 & 0.08 & 0.08 \\
\hline Soybean oil & 0.9 & 0.9 & 0.85 & 0.8 \\
\hline Spirulina & 0 & 0.5 & 1 & 2 \\
\hline Limestone & 1.29 & 1.29 & 1.29 & 1.29 \\
\hline Crude protein (\%) & 18.28 & 18.3 & 18.3 & 18.34 \\
\hline Calcium (\%) & 0.78 & 0.78 & 0.78 & 0.78 \\
\hline Available phosphorous (\%) & 0.38 & 0.38 & 0.38 & 0.39 \\
\hline DL-methionine $(\%)$ & 0.34 & 0.34 & 0.34 & 0.34 \\
\hline Lysine (\%) & 0.86 & 0.86 & 0.86 & 0.86 \\
\hline Methionine+cysteine (\%) & 0.67 & 0.67 & 0.67 & 0.67 \\
\hline
\end{tabular}

${ }^{1)}$ Vitamin premix contained per kilogram: vitamin $A, 9,000 \mathrm{IU}$; vitamin $\mathrm{D}_{3}, 2,000 \mathrm{IU}$; vitamin $\mathrm{E}, 18 \mathrm{IU}$; vitamin $\mathrm{K}_{3}, 2 \mathrm{~g}$; vitamin $\mathrm{B}_{1}, 1.8 \mathrm{mg}$; vitamin $\mathrm{B}_{2}, 6.6 \mathrm{mg}$; vitamin $\mathrm{B}_{3}, 30$ mg; vitamin $B_{4}, 0.1 \mathrm{mg}$; vitamin $B_{5}, 10$ mg; vitamin $B_{6}, 3$ mg; vitamin $B_{9}, 1$ mg; vitamin $B_{12}, 0.015$ mg; choline chloride, $500 \mathrm{mg}$.

2) Mineral premix contained per kilogram: $\mathrm{Mn}, 100 \mathrm{mg} ; \mathrm{Fe}, 50 \mathrm{mg} ; \mathrm{Zn}, 100 \mathrm{mg} ; \mathrm{Cu}, 10 \mathrm{mg} ; \mathrm{I}, 1 \mathrm{mg} ; \mathrm{Se}, 0.2 \mathrm{mg}$.

mination of $\mathrm{H} / \mathrm{L}$, fresh blood smears were prepared and after fixing with methanol, were stained with Giemsa stain in a Coplin jar. A total of 60 cells were counted for each blood slide by using of a light microscope [6].

\section{Statistical analysis}

The general linear model procedure was used to analyze data (Cary, NC, USA). The means were compared using Duncan's multiple range test at probability of 0.05 .

\section{RESULTS}

Table 2. The effects of Spirulina platensis (SP) supplement on performance of broiler chickens during 17 to 24 days of age

\begin{tabular}{lcccc}
\hline \multirow{2}{*}{ Experimental groups } & \multicolumn{4}{c}{ Performance parameters (17 to 24 days of age) } \\
\cline { 2 - 5 } & Weight gain $(\mathbf{g})$ & Feed intake $(\mathbf{g})$ & Feed conversion ratio (FCR) & Body weight at 24 days of age (g) \\
\hline Control') $^{1)}$ & 415.6 & 683.8 & 1.64 & 1,027 \\
$0.5 \%$ SP & 408.4 & 713.8 & 1.75 & $1,021.6$ \\
$1 \%$ SP & 408.8 & 710 & 1.73 & $1,023.6$ \\
$2 \%$ SP & 430 & 720.4 & 1.67 & $1,063.6$ \\
Control 2 & 412 & 684.6 & 1.65 & $1,028.8$ \\
SEM & 0.288 & 0.288 & 0.288 & 0.288 \\
p-value & 0.36 & 0.08 & 0.06 & 0.13 \\
\hline
\end{tabular}

SEM, standard error of the mean.

1) Controls 1 and 2 did not receive feed with SP.

\section{Broiler performance}

The addition of Spirulina supplement had no significant effect on weight gain, feed intake and FCR at weekly recording during 17 to 38 days of age (Tables 2, 3, 4). Under the condition of the present study, final body weight, weight gain, feed intake and FCR did not vary significantly between ConP and ConN after heat challenge at $\mathrm{p}<0.05$. However weight gain between two controls tended to show statistically significant difference $(p=0.06)$. Weight gain was numerically higher in broilers fed $1 \%$ and $2 \%$ Spirulina among heat- exposed birds. Heat- exposed broiler chickens with $0.5 \%$ Spirulina supplement in the diet had the lowest final body weight as compared 
Table 3. The effects of Spirulina platensis (SP) supplement on performance of broiler chickens during 24 to 31 days of age

\begin{tabular}{lcccc}
\hline \multirow{2}{*}{ Experimental groups } & \multicolumn{4}{c}{ Performance parameters (24 to 31 days of age) } \\
\cline { 2 - 4 } & Weight gain (g) & Feed intake (g) & Feed conversion ratio (FCR) & Body weight at 31 days of age (g) \\
\hline Control) $^{1)}$ & 547.6 & 967.6 & 1.76 & $1,574.6$ \\
$0.5 \%$ SP & 535.2 & $1,016.2$ & 1.91 & $1,556.8$ \\
$1 \%$ SP & 535.6 & 937.6 & 1.74 & $1,559.2$ \\
$2 \%$ SP & 568.4 & 970.4 & 1.7 & 1,632 \\
Control 2 & 552.2 & 967.2 & 1.74 & 1,573 \\
SEM & 0.288 & 0.288 & 0.288 & 0.288 \\
p-value & 0.62 & 0.25 & 0.23 & 0.19 \\
\hline
\end{tabular}

SEM, standard error of the mean.

1) Controls 1 and 2 did not receive feed with SP.

Table 4. The effects of Spirulina platensis (SP) supplement on performance of broiler chickens during 31 to 38 days of age

\begin{tabular}{|c|c|c|c|c|}
\hline \multirow{2}{*}{ Experimental groups } & \multicolumn{4}{|c|}{ Performance parameters ( 31 to 38 days of age) } \\
\hline & Weight gain (g) & Feed intake (g) & Feed conversion ratio (FCR) & Body weight at 38 days of age $(\mathrm{g})$ \\
\hline (ontrol') 1 & 569 & $1,153.2$ & 2.05 & $2,143.6$ \\
\hline $0.5 \% \mathrm{SP}$ & 567.6 & $1,161.2$ & 2.09 & $2,124.4$ \\
\hline $1 \% S P$ & 535.2 & $1,185.2$ & 2.25 & $2,094.4$ \\
\hline $2 \% S P$ & 535.2 & $1,218.4$ & 2.30 & $2,167.2$ \\
\hline Control 2 & 570.6 & $1,180.8$ & 2.06 & $2,143.6$ \\
\hline SEM & 0.288 & 0.288 & 0.288 & 0.288 \\
\hline $\mathrm{p}$-value & 0.76 & 0.09 & 0.52 & 0.51 \\
\hline
\end{tabular}

SEM, standard error of the mean.

1) Controls 1 and 2 did not receive feed with SP.

to other treatments $(\mathrm{p}<0.05)$. Broiler chickens in ConN had the lowest FCR among treatments which was not significantly different from those of heat- exposed broilers with $1 \%$ Spirulina in the diet $(\mathrm{p}<0.05)($ Table 5).

\section{Antibody titer against SRBC}

Spirulina supplementation of the diet had significant effects on antibody titer against SRBC (Table 6). At day 38, the anti-

Table 5. The effects of Spirulina platensis (SP) supplement on performance of broiler chickens under high ambient temperature

\begin{tabular}{|c|c|c|c|c|}
\hline \multirow[b]{2}{*}{$\begin{array}{l}\text { Experimental } \\
\text { groups }\end{array}$} & \multicolumn{4}{|c|}{ Performance parameters (38 to 44 days of age) } \\
\hline & $\begin{array}{l}\text { Body weight } \\
\text { (g) }\end{array}$ & $\begin{array}{l}\text { Weight gain } \\
\text { (g) }\end{array}$ & $\begin{array}{l}\text { Feed intake } \\
\text { (g) }\end{array}$ & $\begin{array}{c}\text { Feed } \\
\text { conversion } \\
\text { ratio (FCR) }\end{array}$ \\
\hline ConN $^{1)}$ & $2,814^{\mathrm{a}}$ & 640.8 & $1,309.1^{\mathrm{ab}}$ & $2.04^{c}$ \\
\hline $0.5 \% \mathrm{SP}$ & $2,663.5^{c}$ & 539.1 & $1,263.4^{\mathrm{ab}}$ & $2.34^{\mathrm{a}}$ \\
\hline $1 \% \mathrm{SP}$ & $2,729.3^{b c}$ & 574.6 & $1,180.5^{b}$ & $2.06^{b c}$ \\
\hline $2 \% \mathrm{SP}$ & $2,769.4^{\mathrm{ab}}$ & 602.2 & $1,385.2^{\mathrm{a}}$ & $2.3^{\mathrm{ab}}$ \\
\hline ConP $P^{2)}$ & $2,750.2^{\mathrm{ab}}$ & 564.9 & $1,227.8^{b}$ & $2.16^{\mathrm{abc}}$ \\
\hline SEM & 14.748 & 12.12 & 23.318 & 0.041 \\
\hline p-value & 0.01 & 0.06 & 0.04 & 0.04 \\
\hline
\end{tabular}

SEM, standard error of the mean.

1) Negative control, no exposure to high ambient temperature and no SP.

2) Positive control, exposure to high ambient temperature and no SP.

a-c Different superscripts in each column show significant differences at $p<0.05$. body production against SRBC was higher in broiler chickens with $0.5 \%$ and $2 \%$ Spirulina supplementation as compared to the control groups $(\mathrm{p}=0.002)$. Exposure to high ambient temperature significantly decreased antibody titer compared to non-heat- exposed birds (3.8 and 4.1 for exposure to high ambient temperature and non- exposure to high ambient temperature respectively). As shown in Table 6, in spite of negative impact of heat- exposure on humoral immune response, all broilers fed Spirulina had higher antibody titers as compared to $\operatorname{ConP}(\mathrm{p}=0.001)$.

\section{Serum antioxidant status}

Examined serum oxidant/antioxidant variables were significantly affected by Spirulina supplementation of broiler chickens diet (Table 7). Values for GPx at days 35 and 42 were not statistically significant among experimental treatments hence these data are not shown. Before heat exposure, all Spirulinasupplemented broilers had lower MDA values and higher activity of SOD as compared to non-Spirulina fed birds while increased activity of GPx was only evident in $2 \%$ Spirulinasupplemented group $(\mathrm{p}<0.05)$. As expected, heat stress had significant effect on MDA value as well as activities of SOD and GPx, since ConP presented increased MDA value and lower activities of SOD and GPx compared to ConN ( $\mathrm{p}=$ 0.001). As demonstrated in Table 7, 2\% Spirulina- supple- 
Table 6. The effects of Spirulina platensis (SP) supplement on hemaglutination titer against SRBC

\begin{tabular}{lcccccccc}
\hline Parameter & Day & $\mathbf{0 . 5 \%} \mathrm{SP}$ & $\mathbf{1} \% \mathrm{SP}$ & $\mathbf{2} \% \mathrm{SP}$ & ConN $^{1)}$ & ConP $^{2)}$ & SEM & p-value \\
\hline Antibody titer & 38 & $4.76^{\mathrm{a}}$ & $4^{\mathrm{c}}$ & $4.5^{\mathrm{ab}}$ & $4.27^{\mathrm{bc}}$ & $4.23^{\mathrm{bc}}$ & 0.076 & 0.002 \\
$(\log 2)$ & 45 & $4.38^{\mathrm{ab}}$ & $4.76^{\mathrm{a}}$ & $4.7^{\mathrm{a}}$ & $4.1^{\mathrm{bc}}$ & $3.8^{\mathrm{c}}$ & 0.09 & 0.001 \\
\hline
\end{tabular}

SRBC, sheep red blood cells; SEM, standard error of the mean.

1) Negative control, no exposure to high ambient temperature and no SP.

2) Positive control, exposure to high ambient temperature and no SP.

${ }^{a-c}$ Different superscripts in each row show significant differences at $p<0.05$.

Table 7. The effects of Spirulina platensis (SP) supplement on some antioxidant parameters of broiler chickens

\begin{tabular}{|c|c|c|c|c|c|c|c|c|c|c|}
\hline \multirow{2}{*}{ Day } & \multicolumn{4}{|c|}{ Malondialdehyde (nmol/mL) } & \multicolumn{4}{|c|}{ Superoxide dismutase $\left(\mathrm{UE}^{1)} / \mathrm{mL}\right)$} & \multicolumn{2}{|c|}{ Glutathione peroxidase $(\mu \mathrm{m} / \mathrm{mL})$} \\
\hline & 35 & 38 & 42 & 45 & 35 & 38 & 42 & 45 & 38 & 45 \\
\hline $\operatorname{ConN}^{2)}$ & $8.79^{\mathrm{a}}$ & $9.55^{\mathrm{a}}$ & $9.22^{b}$ & $9.1^{\mathrm{c}}$ & $87.54^{c}$ & $88.85^{b c}$ & $95.33^{\mathrm{a}}$ & $94.84^{\mathrm{a}}$ & $2.33^{b}$ & $2.98^{\mathrm{a}}$ \\
\hline $0.5 \% \mathrm{SP}$ & $8.2^{b}$ & $9^{b}$ & $10.67^{\mathrm{a}}$ & $11.06^{\mathrm{a}}$ & $87.92^{c}$ & $90.92^{\mathrm{a}}$ & $80.12^{d}$ & $80.18^{c}$ & $2.33^{b}$ & $1.97^{b}$ \\
\hline $1 \% S P$ & $8.01^{b}$ & $9.34^{\mathrm{ab}}$ & $10.4^{\mathrm{a}}$ & $10.03^{b}$ & $92.38^{\mathrm{b}}$ & $90.47^{\mathrm{ab}}$ & $82.8^{c}$ & $79.91^{c}$ & $2.35^{b}$ & $2.28^{b}$ \\
\hline $2 \% S P$ & $7.19^{c}$ & $8.54^{c}$ & $9.72^{b}$ & $9.54^{\mathrm{bc}}$ & $98.18^{\mathrm{a}}$ & $91.7^{\mathrm{a}}$ & $91.74^{b}$ & $90.31^{b}$ & $2.7^{\mathrm{a}}$ & $2.87^{\mathrm{a}}$ \\
\hline $\operatorname{ConP}^{3)}$ & $8.63^{\mathrm{a}}$ & $9.61^{\mathrm{a}}$ & $10.94^{\mathrm{a}}$ & $10.86^{a}$ & $87.62^{c}$ & $88.18^{c}$ & $79.78^{d}$ & $80.32^{c}$ & $2.24^{b}$ & $2.01^{b}$ \\
\hline SEM & 0.119 & 0.099 & 0.149 & 0.171 & 0.868 & 0.365 & 1.334 & 1.416 & 0.051 & 0.097 \\
\hline$p$-value & 0.001 & 0.002 & 0.001 & 0.001 & 0.001 & 0.003 & 0.001 & 0.001 & 0.03 & 0.001 \\
\hline
\end{tabular}

SEM, standard error of the mean.

1) UE, unit of enzyme, the amount of enzyme capable of inhibiting the rate of pyrogallol oxidation by $50 \%$.

${ }^{2)}$ Negative control, no exposure to high ambient temperature and no SP.

${ }^{3)}$ Positive control, exposure to high ambient temperature and no SP.

${ }^{a-c}$ Different superscripts in each column show significant differences at $p<0.05$.

mented group had significantly lower MDA value and higher activities of SOD and GPx in comparison with other heatexposed groups $(\mathrm{p}<0.05)$.

\section{Lipid profile}

In pre stress phase, feeding broilers with Spirulina especially at $1 \%$ and $2 \%$ significantly reduced the serum levels of cholesterol, triglyceride and total lipid as compared with control (Table 8). Under heat stress condition, broilers fed Spirulina had significantly reduced serum levels of cholesterol, triglyceride and total lipid regardless of inclusion level as compared to ConP ( $p \leq 0.008)$. In this regard, beneficial effects of $1 \%$ and $2 \%$ Spirulina supplementations on reducing triglyceride were identical on day 45. Compared to ConP, broiler chickens fed Spirulina had significantly higher HDL-C level at the end of experiment although this effect was not observed in preceding days.

\section{Stress indicators}

There was significant difference in corticosterone level of various treatments at all sampling times as presented in Table 9. At day 38, the lowest concentration of corticosterone was observed in 2\% Spirulina- supplemented group. Broilers reared under high ambient temperature, had significantly higher corticosterone concentration than those reared under control temperature regardless of Spirulina addition $(\mathrm{p}=0.001)$. Dur-

Table 8. The effects of Spirulina platensis (SP) supplement on lipid profile of broiler chickens

\begin{tabular}{|c|c|c|c|c|c|c|c|c|c|c|c|c|c|c|c|c|}
\hline \multirow{2}{*}{ Item } & \multicolumn{4}{|c|}{ Cholesterol (mg/dL) } & \multicolumn{4}{|c|}{ Triglyceride (mg/dL) } & \multicolumn{4}{|c|}{ High density lipoprotein (mg/dL) } & \multicolumn{4}{|c|}{ Total lipid (mg/dL) } \\
\hline & $35 \mathrm{~d}$ & $38 \mathrm{~d}$ & $42 \mathrm{~d}$ & $45 d$ & $35 \mathrm{~d}$ & $38 \mathrm{~d}$ & $42 d$ & $45 d$ & $35 \mathrm{~d}$ & $38 \mathrm{~d}$ & $42 d$ & $45 d$ & $35 \mathrm{~d}$ & $38 \mathrm{~d}$ & $42 d$ & $45 d$ \\
\hline ConN $^{1)}$ & $162.48^{\mathrm{a}}$ & $169.6^{\mathrm{a}}$ & $144.5^{b}$ & $163.06^{b}$ & $97.36^{\mathrm{a}}$ & $98.34^{\mathrm{ab}}$ & $93.86^{b}$ & $84.62^{b}$ & $72.92^{\mathrm{a}}$ & $69.2^{\mathrm{a}}$ & $56.8^{\mathrm{bc}}$ & $61.19^{a}$ & $336.06^{\mathrm{a}}$ & $352.18^{a}$ & $310.5^{b}$ & $293.7^{b}$ \\
\hline $0.5 \% \mathrm{SP}$ & $160.36^{\mathrm{a}}$ & $160.3^{b}$ & $144.3^{b}$ & $141.24^{\mathrm{bc}}$ & $97.4^{\mathrm{a}}$ & $96.74^{\mathrm{bc}}$ & $93.96^{b}$ & $84.02^{b}$ & $64.05^{\mathrm{ab}}$ & $70.08^{a}$ & $61.72^{\mathrm{ab}}$ & $60.25^{a}$ & $335.9^{\mathrm{a}}$ & $337.1^{b}$ & $310.62^{b}$ & $293.94^{b}$ \\
\hline $1 \% \mathrm{SP}$ & $155.18^{a}$ & $150.3^{c}$ & $141.7^{b}$ & $137.78^{c}$ & $93.99^{b}$ & $95.3^{c}$ & $90.74^{c}$ & $77.12^{c}$ & $63.44^{\mathrm{ab}}$ & $61.35^{\mathrm{b}}$ & $54.78^{\mathrm{bc}}$ & $64.12^{\mathrm{a}}$ & $324.78^{\mathrm{a}}$ & $320.04^{c}$ & $303.78^{c}$ & $280.28^{c}$ \\
\hline $2 \% S P$ & $144.5^{b}$ & $144.64^{d}$ & $128^{c}$ & $126.52^{d}$ & $90.38^{c}$ & $64.92^{d}$ & $86.4^{d}$ & $76.9^{c}$ & $59.24^{b}$ & $59.4^{b}$ & $52.85^{c}$ & $53.21^{b}$ & $306.17^{b}$ & $309.22^{d}$ & $282.03^{d}$ & $265.34^{d}$ \\
\hline ConP ${ }^{2)}$ & $160.6^{\mathrm{a}}$ & $170.2^{\mathrm{a}}$ & $171.1^{\mathrm{a}}$ & $152.4^{\mathrm{a}}$ & $96.94^{\mathrm{a}}$ & $99.86^{\mathrm{a}}$ & $99.26^{a}$ & $92.96^{\mathrm{a}}$ & $72.74^{\mathrm{a}}$ & $68.56^{a}$ & $64.4^{\mathrm{a}}$ & $45.4 c$ & $336.14^{\mathrm{a}}$ & $352.2^{\mathrm{a}}$ & $352.4^{\mathrm{a}}$ & $319.96^{a}$ \\
\hline SEM & 1.837 & 2.143 & 2.908 & 1.782 & 0.631 & 0.615 & 0.937 & 1.261 & 1.936 & 1.234 & 1.273 & 1.564 & 2.84 & 3.648 & 4.697 & 3.846 \\
\hline$p$-value & 0.003 & 0.001 & 0.001 & 0.001 & 0.001 & 0.001 & 0.001 & 0.001 & 0.07 & 0.002 & 0.008 & 0.001 & 0.001 & 0.001 & 0.001 & 0.001 \\
\hline
\end{tabular}

SEM, standard error of the mean.

1) Negative control, no exposure to high ambient temperature and no SP.

2) Positive control, exposure to high ambient temperature and no SP.

a-c Different superscripts in each column show significant differences at $p<0.05$. 
Table 9. The effects of Spirulina platensis (SP) supplement on stress indexes of broiler chickens

\begin{tabular}{lcccccccc}
\hline Parameters & Day & $\mathbf{0 . 5 \% ~ S P}$ & $\mathbf{1 \% ~ S P}$ & $\mathbf{2 \% ~ S P}$ & ConN $^{1)}$ & ConP $^{2)}$ & SEM & p-value \\
\hline $\begin{array}{l}\text { Corticosterone concentration } \\
\text { (nmol/L) }\end{array}$ & 38 & $27.25^{\mathrm{a}}$ & $27.81^{\mathrm{a}}$ & $25.63^{\mathrm{b}}$ & $26.77^{\mathrm{ab}}$ & $27.44^{\mathrm{a}}$ & 0.24 & 0.02 \\
& 42 & $32.19^{\mathrm{b}}$ & $31.87^{\mathrm{b}}$ & $30.04^{\mathrm{c}}$ & $27.15^{\mathrm{d}}$ & $33.38^{\mathrm{a}}$ & 0.465 & 0.001 \\
$\mathrm{H} / \mathrm{L}^{3)}$ ratio & 45 & $34.41^{\mathrm{a}}$ & $32.93^{\mathrm{b}}$ & $30.64^{\mathrm{c}}$ & $28.79^{\mathrm{d}}$ & $35.02^{\mathrm{a}}$ & 0.497 & 0.001 \\
& 35 & $0.297^{\mathrm{b}}$ & $0.402^{\mathrm{a}}$ & $0.196^{\mathrm{c}}$ & $0.358^{\mathrm{ab}}$ & $0.354^{\mathrm{ab}}$ & 0.016 & 0.001 \\
& 45 & $0.414^{\mathrm{c}}$ & $0.54^{\mathrm{b}}$ & $0.405^{\mathrm{c}}$ & $0.569^{\mathrm{b}}$ & $0.709^{\mathrm{a}}$ & 0.025 & 0.001 \\
\hline
\end{tabular}

SEM, standard error of the mean.

1) Negative control, no exposure to high ambient temperature and no SP

2) Positive control, exposure to high ambient temperature and no SP

3) Heterophil to Lymphocyte ratio.

${ }^{a-c}$ Different superscripts in each row show significant differences at $p<0.05$.

ing heat stress, broilers fed Spirulina especially at $1 \%$ or $2 \%$ of the diet had significantly lower corticosterone concentration as compared to ConP. Similar results were found for $\mathrm{H} / \mathrm{L}$ ratio. Acute heat stress significantly increased $\mathrm{H} / \mathrm{L}$ ratio as compared to non-heat-stressed birds (ConN). The H/L ratio was significantly lower in all broilers fed with Spirulina under high ambient temperature as compared to their heat- exposed but no Spirulina supplemented counterparts (ConP).

\section{DISCUSSION}

Exposure of broiler chickens to high ambient temperature has well recognized negative consequences on the poultry industry worldwide. Heat stress has noticeable detrimental effect on production performance by impairment of nutrient utilization and digestibility. The activities of enzymes of protein digestion decrease significantly with a high ambient temperature [2]. Khosravinia [7] reported that addition of phytogenic products into the drinking water of heat- stressed broiler chickens improves production efficiency of poultry by promoting digestion process and creating slight improvement in FCR. In the current study although stress in heat- exposed birds were physiologically evident, the challenge was unable to exert significant impairment in performance characteristics. An explanation for this finding is that since at the time that the experiment was done the climate was hot and the temperature of the growing house during first 17 days was inevitably higher than what is recommended, birds might acquire some heat adaptation. However mean weight gain values in ConP and ConN tended to show the statistically noticeable difference $(p=0.06)$. In the present study, Spirulina failed to affect growth performance in pre or post stress periods. These results are in agreement with those obtained by Mariey et al [10] who reported no adverse effect of Spirulina supplementation on body weight of growing pullets or laying hens. Also, Canogullari Dogan et al [21] reported that feeding of laying Japanese quails with Spirulina platensis addition did not significantly affect FCR and feed intake. However Kharde et al [22] reported that feeding of broiler chickens on diet supplemented with Spirulina at 300 or $500 \mathrm{mg} / \mathrm{kg}$ feed for six weeks significantly increased the mean body weight, weight gain and feed efficiency compared to control group. Shanmugapriya et al [23] reported significant decrease in FCR by the dietary inclusion of the $1 \%$ of Spirulina platensis as compared to the control broilers. In a study by Kaoud [24], it has been shown that FCR was lower for broiler chickens supplemented with Spirulina platensis than control birds or birds supplemented with prebiotics.

Certain species of Spirulina including Spirulina platensis have exhibited immunomodulating properties in animals and human [25]. In the current study, at day 45 , the lowest value for HA titer against SRBC antigen occurred in heat- stressed with no Spirulina supplementation (ConP). Our study revealed that all Spirulina supplemented groups showed significantly higher secondary antibody response against SRBC antigen despite heat stress conditions when compared with ConP. The result of the current study was in agreement with the Qureshi et al [26] who indicated a positive effect on second humoral response against SRBC antigen in broiler chickens fed with Spirulina compared to the control.

Heat stress stimulates oxidative damages of tissues by increasing the release of corticosterone and triggering lipid peroxidation in cell membranes [1]. In heat-challenged broilers, the increased level of MDA as well as decreased activities of serum SOD and GPx as reported earlier imply an imbalance in the oxidants/antioxidants system and oxidative stress [27]. Hence, organic compounds that interfere with free radicals may restore the balance of oxidants/antioxidants and leading to the improvement of health and growth. Spirulina platensis contains C-phycocyanin, a potent antioxidant agent as one of its major constituents [28]. Accordingly, in the current study heat-exposed broiler chickens with 2\% Spirulina in the diet presented significantly lower MDA level and higher SOD and GPx activities as compared with other heat- exposed birds. Increased activities of SOD and GPx enzymes may improve the state of antioxidant defense system of broiler chickens. In agreement with a previous report which indicated dose-dependent anti-inflammatory effect of C-phycocyanin in some laboratory animals [29], our data presented the dose- depen- 
dency of antioxidant effect for Spirulina supplement in broiler chickens. Akbarian et al [30] reported that some antioxidant phytochemicals, such as flavonoids and related compounds, were shown to decrease MDA value and induce elevation of SOD in heat- stressed poultry while having less or no effect in non-heat- stressed birds. Our results revealed that Spirulina supplementation enhanced antioxidant status of the body during pre heat stress at 38 days of age by increasing the activity of SOD and GPx and reducing MDA level. Therefore, our results support that unlike some phytochemicals, Spirulina antioxidant components have the potential to exert beneficial effects under both normal and challenging environmental conditions. This is consistent with a previous finding by ELSabagh et al [15] who reported lower serum MDA in fattening lambs fed with Spirulina compared to the control. Protective effects of Spirulina supplementation against oxidative damage have also been reported in laboratory animals [12].

The hypolipidemic effect of Spirulina or its extracts have been determined by various experiments on laboratory animals $[11,12]$. In the present study, our data revealed significant reductions in serum cholesterol, triglyceride and total lipid in pre or post stress periods as the level of dietary Spirulina was increased. However, raising activity of Spirulina supplement on HDL-C level was evident only on day 45 where heat-stressed broilers with dietary Spirulina showed significantly higher HDL-C than ConP. This is in consistent with previous finding by Canogullari Dogan et al [21] who reported that HDL cholesterol concentration increased with the increased supplementation of Spirulina platensis. Spirulina platensis supplementation also decreased plasma total cholesterol and trigyliceride levels between groups numerically but not statistically. In a study by Hosseini-Vashan et al [31], blood total cholesterol level in broiler chickens pre or after heat stress could be decreased by feeding them with a phytogenic compound. Mariey et al [10] by evaluating the effects of Spirulina platensis on local laying hens observed that plasma cholesterol were significantly decreased in birds fed with the Spirulinacontaining diets as compared to the control. Hypolipidemic effect of Spirulina has been reported to be due to the C-phycocyanin which inhibits the pancreatic lipase activity in a dosedependent manner [11].

Corticosterone concentration which is elevated under stimulation of stress factors such as high ambient temperature induces disappearing of lymphocytes and leading to lymphopenia [8]. Regardless of inclusion rate, supplementation of the diet with Spirulina resulted in significantly lower $\mathrm{H} / \mathrm{L}$ ratio as compared to ConP. This observation may be partly due to the antioxidant property of Spirulina which leads to membrane stabilization and mostly because of declined secretion of corticosterone.

In conclusion, our results showed that supplementation of the broiler chicken's diet with Spirulina had beneficial effect in reducing heat stress- induced hyperlipaemia and oxidative stress with no adverse effect on performance. Supplementary Spirulina also exhibited immune enhancing effects by increasing the humoral antibody response. Therefore, the present study for the first time suggests that alleviation of biochemical adverse impacts due to high ambient temperature in broiler chickens may be approached through supplementation of the diet with Spirulina platensis. More studies with Spirulina supplement for other animal species under heat stress or any other environmental suboptimal conditions in future researches will provide further insights regarding its indications and usefulness.

\section{CONFLICT OF INTEREST}

We certify that there is no conflict of interest with any financial organization regarding the material discussed in the manuscript.

\section{ACKNOWLEDGMENTS}

The authors are thankful to all the staffs in animal science research institute, Alborz province, Iran. Technical supports by F. Yari and M. Bagheri were gratefully appreciated.

\section{REFERENCES}

1. Mujahid A, Akiba Y, Warden CH, Toyomizu M. Sequential changes in superoxide production, anion carriers and substrate oxidation in skeletal muscle mitochondria of heat-stressed chickens. FEBS Lett 2007;581:3461-7.

2. Sahin K, Kucuk O, Sahin N, Sari M. Effects of vitamin C and vitamin $\mathrm{E}$ on lipid peroxidation status, serum hormone, metabolite and mineral concentrations of Japanese quails reared under heat stress (34 degrees C). Int J Vitam Nutr Res 2002; 72:91-100

3. Lin H, Wang LF, Song JL, Xie YM, Yang QM. Effect of dietary supplemental levels of vitamin A on the egg production and immune responses of heat-stressed laying hens. Poult Sci 2002; 81:458-65.

4. Ahmed T, Sarwar M. Dietary electrolyte balance: implications in heat stressed broilers. Worlds Poult Sci J 2006;63:638-53.

5. Zulkifli IN, Abdullah NM, Azrin M, Ho YW. Growth performance and immune response of two commercial broiler strains fed diets containing Lactobacillus cultures and oxytetracycline under heat stress conditions. Br Poult Sci 2000;41:593-7.

6. Houshmand M, Azhar K, Zulkifli I, Bejo MH, Kamyab A. Effects of prebiotic, protein level, and stocking density on performance, immunity, and stress indicators of broilers. Poult Sci 2012;91:393-401.

7. Khosravinia H. Mortality, production performance, water intake and organ weight of the heat stressed broiler chicken 
given savory (Satureja khuzistanica) essential oils through drinking water. J Appl Anim Res 2016;44:273-80.

8. Abbas AO, Gehad AE, Hendricks GL, Gharib HBA, Mashaly MM. The effect of lighting program and melatonin on the alleviation of the negative impact of heat stress on the immune response in broiler chickens. Int J Poult Sci 2007;6:651-60.

9. Farag MR, Alagawany M, Abd El-Hack ME, Dhama K. Nutritional and healthical aspects of Spirulina (Arthrospira) for poultry, animals and human. Int J Pharmacol 2016;12:36-51.

10. Mariey YA, Samak HR, Ibrahem MA. Effect of using Spirulina platensis algae as a feed additive for poultry diets: 1- productive and reproductive performances of local laying hens. Egypt Poult Sci 2012;32:201-15.

11. Deng R, Chow TJ. Hypolipidemic, antioxidant, and antiinflammatory activities of microalgae Spirulina. Cardiovasc Ther 2010;28:e33-45.

12. Bashandy SA, Alhazza IM, El-Desoky GE, Al-Othman ZA. Hepatoprotective and hypolipidemic effects of Spirulina platensis in rats administered mercuric chloride. Afr J Pharm Pharmacol 2011;5:175-82.

13. Holman BWB, Malau-Aduli AEO. Spirulina as a livestock supplement and animal feed. J Anim Physiol Anim Nutr 2013; 97:615-23.

14. Zahroojian N, Moravej H, Shivazad M. Effects of dietary marine algae (Spirulina platensis) on egg quality and production performance of laying hens. J Agric Sci Technol 2013;15:1353-60.

15. EL-Sabagh MR, Abd-Eldaim MA, Mahboub DH, Abdel-Daim M. Effects of Spirulina platensis algae on growth performance, antioxidative status and blood metabolites in fattening lambs. J Agric Sci 2014;6:92-8.

16. Zeweil H, Abaza IM, Zahran SM, et al. Effect of Spirulina platensis as dietary supplement on some biological traits for chickens under heat stress condition. Asian J Biomed Pharm Sci 2016;6(56):8-12.

17. NRC. Nutrient requirements of poultry. Ninth Revised Edition. Washington, DC, USA: National Academy Press; 1994.

18. Ali OH, Elzubeir EA, Elhadi HM. Effect of season on the immunity of newly hatched broiler chicks reared in arid-hot climate. Pak J Biol Sci 2008;11:318-20.

19. Paglia DE, Valentine WN. Studies on the quantitative and qualitative characterization of erythrocyte glutathione peroxi- dase. J Lab Clin Med 1967;70:158-69.

20. Marklund S, Marklund G. Involvement of superoxide anion radical in autoxidation of pyrogallol and a convenient assay of superoxide dismutase. Eur J Biochem 1974;46:469-74.

21. Canogullari Dogan S, Baylan M, Erdogan Z, et al. Performance, egg quality and serum parameters of Japanese quails fed diet supplemented with Spirulina platensis. Fresen Environ Bull 2016;25:5857-62.

22. Kharde SD, Shirbhate RN, Bahiram KB, Nipane SF. Effect of Spirulina supplementation on growth performance of broilers. Indian J Vet Res 2012;21:66-9.

23. Shanmugapriya B, Babu SS, Hariharan T, Sivaneswaran S, Anusha MB. Dietary administration of Spirulina platensis as probiotics on growth performance and histopathology in broiler chicks. Int J Recent Sci Res 2015;6:2650-3.

24. Kaoud AH. Effect of Spirulina platensis as a dietary supplement on broiler performance in comparison with prebiotics. Spec J Biol Sci 2015;1:1-6.

25. Khan Z, Bhadouria P, Bisen PS. Nutritional and therapeutic potential of Spirulina. Curr Pharm Biotechnol 2005;6:373-9.

26. Qureshi MA, Garlich JD, Kidd MT. Dietary Spirulina platensis enhances humoral and cell-mediated immune functions in chickens. Immunopharmacol Immunotoxicol 1996;18:465-76.

27. Georgieva NV, Koinarsk V, Gadjeva V. Antioxidant status during the course of Eimeria tenella infection in broiler chickens. Vet J 2006;172:488-92.

28. Bhat VB, Madyastha KM. C-phycocyanin: a potent peroxyl radical scavenger in vivo and in vitro. Biochem Biophys Res Commun 2000;275:20-5.

29. Romay C, Ledón N, González R. Further studies on antiinflammatory activity of phycocyanin in some animal models of inflammation. Inflamm Res 1998;47:334-8.

30. Akbarian A, Michiels J, Degroote J, et al. Association between heat stress and oxidative stress in poultry; mitochondrial dysfunction and dietary interventions with phytochemicals. J Anim Sci Biotechnol 2016;7:37.

31. Hosseini-Vashan SJ, Golian A, Yaghobfar A, et al. Antioxidant status, immune system, blood metabolites and carcass characteristic of broiler chickens fed turmeric rhizome powder under heat stress. Afr J Biotechnol 2012;11:16118-25. 